\title{
Landnutzungskarte - Ergebnis einer halbautomatischen Auswertung von Falschfarbenluftbildern
}

\section{Einleitung}

Ziel und Zweck der Luftbildinterpretation gipfeln fast immer in einer Kartierung des Bildinhaltes. Im vorliegenden Beispiel galt es die Landnutzung (im engeren Sinne die landwirtschaft lichen Kulturen) aus Luftbildern zu kartieren. Dass für diese Aufgabenstellung FalschfarbenAufnahmen besonders geeignet sind, liegt an der Empfindlichkeit des Falschfarbenfilms im Reflexionsbereich der Vegetation. Beim verwendeten Bildmaterial (Abb. 1) handelt es sich um Falschfarbendiapositive mit folgenden Merkmalen: Bildmassstab 1 : 8600; Bildformat $23 \times 23$ $\mathrm{cm}$; Senkrechtaufnahmen; Aufnahmedatum 4. Juni 1964; Flugzeit 1330 Uhr; Flughöhe ca. $1000 \mathrm{~m}$ über Grund; Objektiv UAg (Universal-Aviogon) der Firma Wild Heerbrugg mit einer Brennweite von $152 \mathrm{~mm}$; Aufnahmegebiet Unterstammheim Kanton Zürich.

Das Stammheimertal wurde als Testgebiet gewählt, weil hier auf engem Raum viele verschiedene landwirtschaftliche Kulturen angebaut werden und im flachen Gelände die Reflexverhältnisse nicht noch zusätzlich durch ein bewegtes Relief belastet werden.

\section{Bildauswertung}

Die Bildauswertung stuitzt sich auf Farbmessungen, die auf dem Computer der Universität Zürich verarbeitet wurden. Eine Luftbildinterpretation, die auf reproduzierbaren Messwerten basiert, ist unabhängig von der subjektiven Beurteilung des Interpreten und eröffnet die Perspektiven einer automatischen Bildverarbeitung.

Der Vorgang der Bildmessung und der Datenverarbeitung sei im Folgenden nur kurz gestreift und im Uebrigen auf die Fachliteratur verwiesen.

Es wurde ein Bildausschnitt von $5 \times 14 \mathrm{~cm}$ bearbeitet, um die zu bewältigende Datenmenge in Grenzen zu halten. Dies entspricht einer Testfläche im Gelände von $430 \times 1204$ m oder von $517720 \mathrm{m2}$. Dieser Ausschnitt wurde mit einem Messgerät des Photographischen Institutes der ETH (Optronics) in 25 u Schritten in allen drei Farbbereichen rot, grün und blau abgetastet. Im Folgenden beschränken wir uns auf die Dichtemessungen im Rotbereich, obwohl durch die Berücksichtigung der beiden übrigen Farbbereiche die Resultate, allerdings mit dreifachem Aufwand, noch verbessert werden könnten. Der gewählte Bildausschnitt (Abb. 1) wurde durch eine
Matrix von 2000 x 5600 Dichtewerten im Rotbereich dargestellt, das sind total 11,2 Mio Pixel (picture elements). Von jeder vorkommenden Landnutzungsart wurde eine kleine Stichprobe herausgesucht (Tab. 1), um aufgrund dieser Werte die Landnutzungstypen des Testgebietes klassieren und kartieren zu können.

Im nächsten Schritt wurden aus den Stichprobendaten und der gesamten Datenmatrix Bildparameter berechnet: Im vorliegenden Fall nur die mittlere Dichte und die Standardabweichung von jeweils $20 \times 20$-Teilmatrizen (im Gelände 4,3 $\times 4,3 \mathrm{~m})$. Mit Hilfe des hier nicht weiter erläuterten, statistischen Verfahrens der Diskriminanzanalyse wurden aus den Stichprobenparametern Trennfunktionen errechnet, mit denen dann alle ermittelten Bildparameter einer bestimmten Landnutzungsart zugeordnet wurden.

$\begin{array}{lrc}\text { Asphaltstrasse } & 222 \mathrm{~m} 2 & - \\ \text { Bahnlinie } & 111 \mathrm{~m} 2 & - \\ \text { Hausdächer } & 222 \mathrm{~m} 2 & - \\ \text { Obstbäume } & 129 \mathrm{~m} 2 & \text { (blank) } \\ \text { Kunstwiesen } & 1025 \mathrm{~m} 2 & + \\ \text { Hopfen } & 721 \mathrm{~m} 2 & = \\ \text { Höckerbohnen } & 647 \mathrm{~m} 2 & \mathrm{e} \\ \text { Tabak } & 184 \mathrm{~m} 2 & : \\ \text { Mais } & 407 \mathrm{~m} 2 & \mathrm{~m} \\ \text { Weizen } & 1682 \mathrm{~m} 2 & \mathrm{w} \\ \text { Sommergerste } & 203 \mathrm{~m} 2 & \mathrm{x} \\ \text { Hafer } & 222 \mathrm{~m} 2 & 0 \\ \text { Wintergerste } & 203 \mathrm{~m} 2 & \mathrm{v} \\ \text { Kartoffeln } & 665 \mathrm{~m} 2 & \mathrm{z} \\ \text { Runkelrüben } & 185 \mathrm{~m} 2 & \mathrm{r} \\ \text { Spinat } & 222 \mathrm{~m} 2 & \mathrm{~s}\end{array}$

Tab. 1: Stichprobenflächen der einzelnen Landnutzungstypen; total 1,36\% des gesamten Testgebietes. Die Symbole beziehen sich auf Abb. 2 .

\section{Kartierung}

Jeder Landnutzungstyp wurde durch ein BuchstabenSymbol dargestellt, sodass die klassifizierte Datenmatrix bereits einer ersten Landnutzungskarte gleichzusetzen ist. Jedes Symbol entspricht in Wirklichkeit einer Fläche von 4,3 $\mathrm{x}$ 4,3 m (= 18,49 m2) im Gelände. Die so erhalteDr. Hans Maurer u. Dr. Guido Dorigo, Geographisches Institut der Universität, Blümlisalpstr. 10, 8006 Zürich 


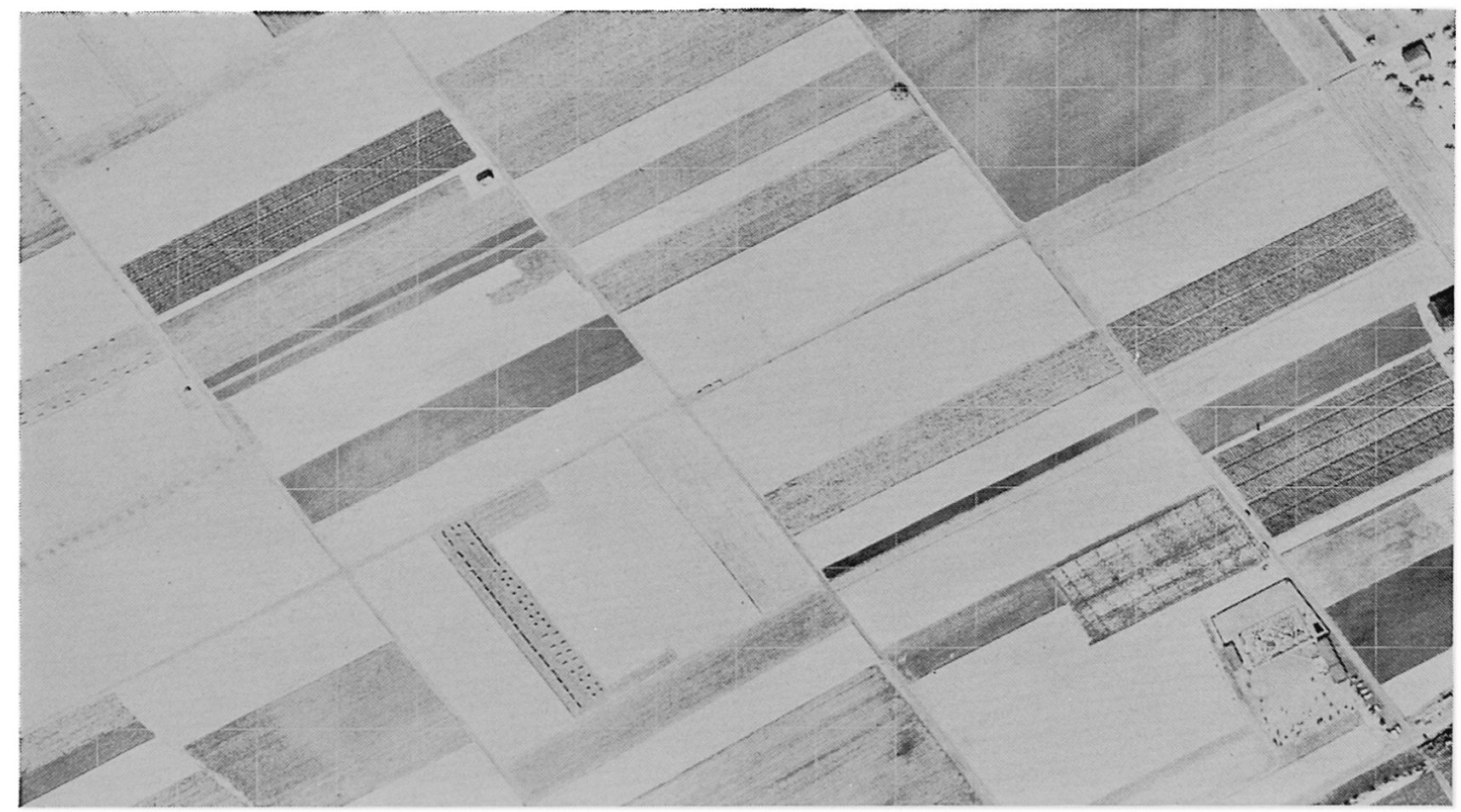

Abb. 1: Rotauszug aus einer Falschfarben-Luftaufnahme

ne Landnutzungskarte hat den Vorteil, dass nében der Darstellung der einzelnen Anbauzonen, durch einfaches Auszählen der Symbole, sofort auch deren Fläche ermittelt werden kann. Dies würde allerdings eine fehlerfreie Klassifizierung voraussetzen.

In der vorliegenden Karte (Abb. 2), welche sich nur auf die Messwerte im Rotbereich und die beiden erwähnten Bildparameter stuitzt, entsprechen die Symbole recht gut der Wirklichkeit. Relativ viele der Fehlklassierungen gehen auf die Verwechslung von Getreidesorten zurück. So wurde
Weizen (w) zu dieser Jahreszeit häufig als Hafer (o) oder Sommergerste (x) klassiert. Oft wurden auch Wiesen mit hohem, reifem Gras als Wintergerste ( $v$ ) angegeben, die zur fraglichen Zeit kurz vor ihrer Ernte stand. Selbstverständlich ist, dass längs der Grenzlinien zwischen zwei Kulturen, bei einem Raster von 4,3 x 4,3 m pro Symbol, Fehlklassierungen entstehen müssen, denn die Grenzrasterflächen haben ja Anteil an beiden benachbarten Feldern. Trotzdem kommen ganz schmale Aecker, wie sie bei Mais oder Runkelrüben vorkommen, z:T. recht gut zum Vorschein.

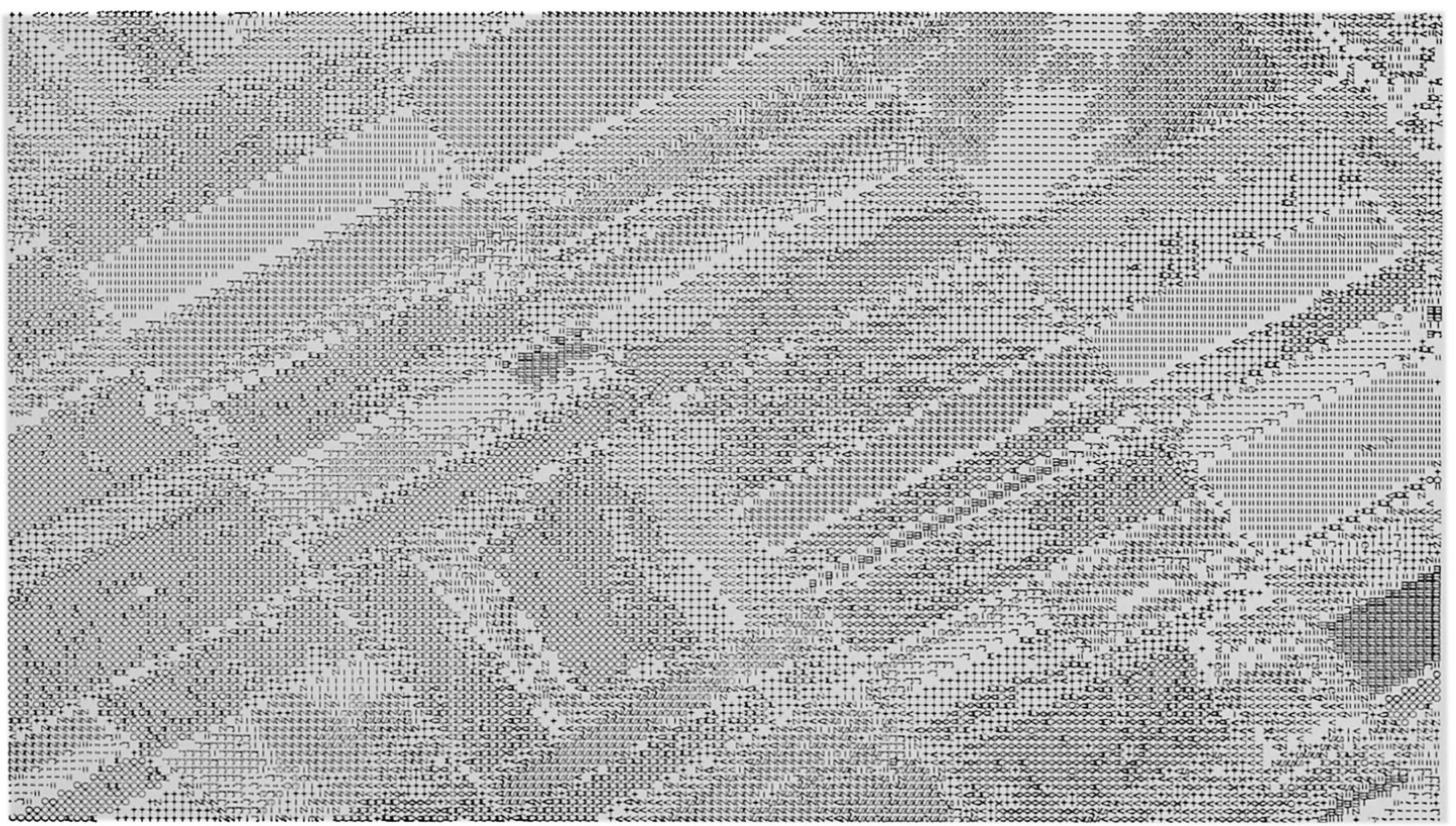

Aibb. 2: Computerkarte der Landnutzung. Symbole siehe Tab. 1 


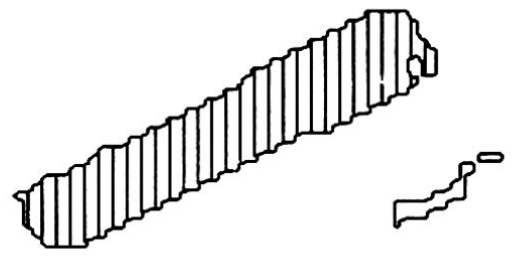

0

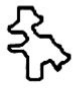

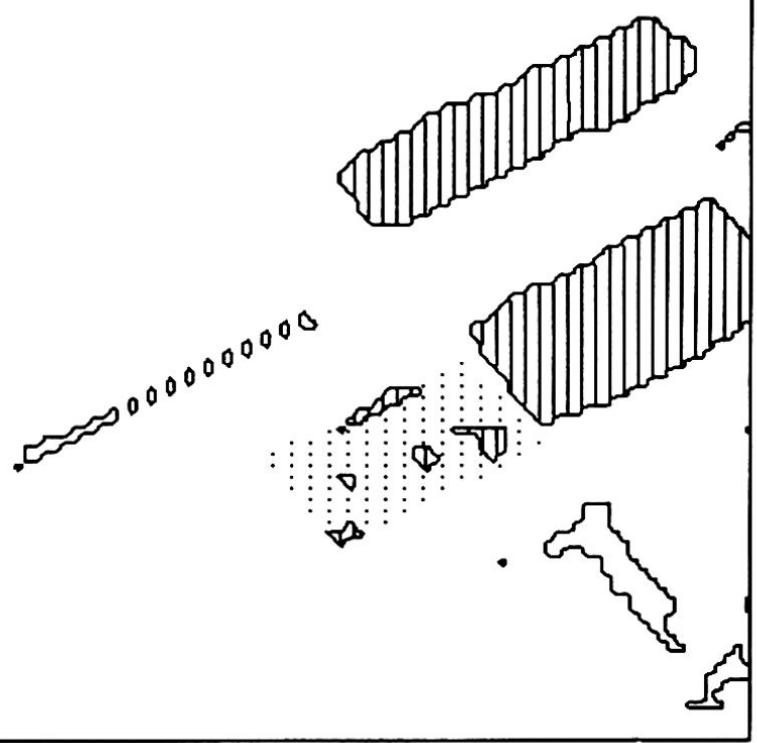

Abb. 3: Hopfenkarte; schraffiert: richtig klassiert

punktiert: sollte Hopten sein

Durch den hohen Anteil an Schatten, wie sie bei Hopfen oder Obstbäumen typisch sind, werden häufig besiedeltes Gebiet oder die Grenzen zwischen den Kulturen als Hopfen ( $=$ ) oder Obstbaum (blank) klassifiziert. Der Vergleich von Abb. 1 mit Abb. 2 zeigt, wie gut die Computerkarte die Wirklichkeit wiederspiegelt. Fehlklassierungen werden verständlich, wenn man die Unregelmässigkeiten im Foto beachtet (Schwimmbad etc.).

Es ist durchaus begreiflich, dass eine relativ frisch geschnittene Wiese mit vielen krautigen Pflanzen, wie Sauerampfer und Löwenzahl als
Acker mit Spinat oder Runkelrüben klassifiziert wird. Viele der oben erwähnten Fehlklassierungen wären da auch von einem guten Interpreten kaum zu vermeiden, denn die verschiedenen Getreidesorten sehen im Luftbild zum Verwechseln ähnlich aus.

Durch ein sog. Smoothing-Programm können einzelne isolierte Fehlklassierungen innerhalb eines Feldes ausgeglättet werden. Abb. 3 und Abb. 4 zeigen, wie aus einer derart korrigierten Matrix eine gewünschte landwirtschaftliche Kultur (im vorliegenden Fall Hopfen und Kartoffeln) durch

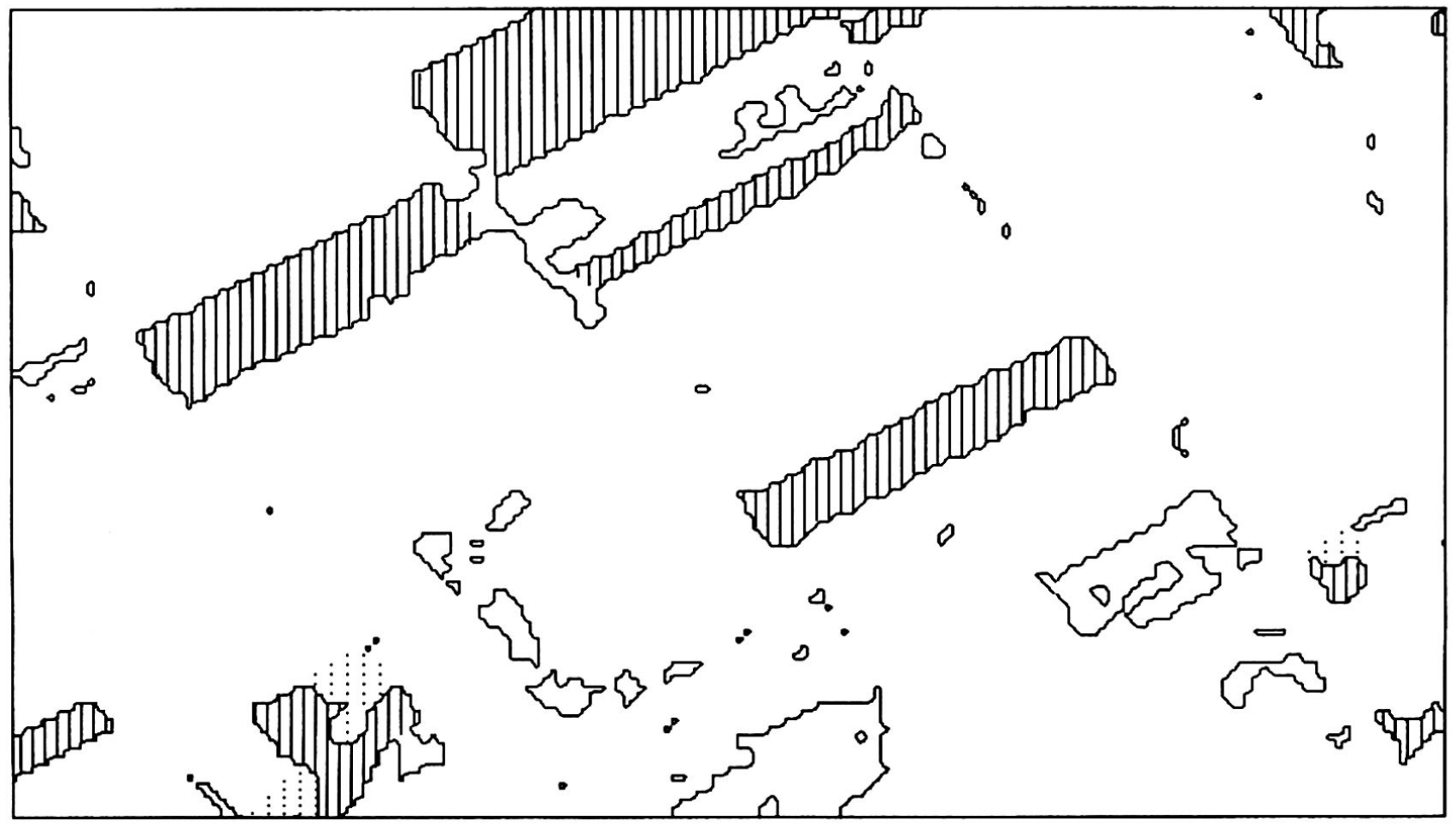

Abb. 4: Kartoffelkarte 
ein Isolinien-Programm mit entsprechender Plotroutine leicht und sauber herausgezeichnet werden kann.

\section{Literatur}

Lintz J. and Simonet D.S., 1976 Remote Sensing for Environment, Addison-Wesley Publishing Company
Maurer H., 1974 Quantification of TexturesTextural parameters and their significance for classifying agricultural crop types from colour aerial photographs. Photogrammetria 30, p.21 - 40

Tomlinson F., 1972 Geographical Data Handling, Symp. Edition Int'1. Geographical Union Commission, Ottawa, Canada.

\section{Literaturbesprechung}

LEWIS Peter, Maps and Statistics, $318 \mathrm{~S}$., div. Tab. u. Fig., Methuen \& Co. Ltd., London 1977 , $£$ 9.50/ paper back $£ 5.95$.

Die Reihe "The Field of Geography" vermittelt mit diversen Textbüchern Aspekte der "neuen" Geographie. Der Student ist heute kaum mehr in der Lage, die zahllosen Publikationen laufend zu verarbeiten. Kompetente Autoren sollen ihm daher zu verschiedenen Themen ein Spektrum der Arbeitstechniken und -konzepte geben. LEWIS versucht in "Maps and Statistics" Verfahren der nichtparametrischen Statistik an der Verarbeitung von Karten zu erläutern. Was in einer Karte auch immer dargestellt sein mag, im Zentrum steht die räumliche Beziehung der punkt- oder linienförmigen Symbole. Der Autor will ein Bindeglied schlagen zwischen Karte - Behauptung
(=Beziehung) - Hypothese und dem Testen dieser Hypothese. Das Werk soll dem Studenten ein elementarer Einführungstext sein. Er brauche nur die Kenntnisse der Schulalgebra - und die Bereitschaft, dieses Wissen wieder etwas aufzufrischen.---Letzteres sollte besonders betont werden. Das Textbuch setzt für ein sauberes Studium doch recht umfangreich mathematisches Denken voraus. Auch die Verbindung Statistik-Karte wurde mehr auf Kosten der Verständlichkeit erzielt. Meiner Meinung nach ist das Lehrziel leichter zu erreichen, wenn der Student Kapitel eines reinen Lehrbuches der nichtparametrischen Statistik durcharbeitet. In einem Universitätskurs könnten dazu parallel (zur Vertiefung der theoretischen Kenntnis) Beispiele aus der Geographie erläutert werden.

GH $1 / 1978$

Guido Dorigo
MAUDER Karl, Landnutzung im Nordost-Transwaal, 314 S. : 1 Kartenband, Regensburger Geogr. Schriften Heft 8, 1976.

Der Verfasser ist Schriftleiter der Regensburger Geogr. Schriften. Die Feldaufnahmen für seine Dissertation entstanden bei seinem Aufenthalt in Südafrika 1959 - 61. An die Uebersicht über Landschaft und Erschliessung des NordostTransvaal schliessen sich 30 Fallstudien von Einzelfarmen der Weissen, die nach ihrer vorherrschenden Landnutzung typisiert werden. Aus diesen Farm-Monographien werden ihre räumliche Abhängigkeit, das sozio-ökonom. Gefüge, die Fragen der Arbeitskräfte und der Technisierung abgeleitet. Der Farmwirtschaft der Weissen wird die Landnutzung in zwei Bantugebieten gegenübergestellt. 95 meist statistische Tabellen und 23 Abbildungen (vorwiegend Kartenskizzen) ergänzen den mit zahlreichen Quellenangaben durchsetzten Text. Der Kartenband enthält eine farbige Höhenstufenkarte als Uebersicht und die Nutzungskarten der aufgenommenen Farmen.
Die umfangreiche und sehr gründliche Untersuchung vermittelt hervorragende Einblicke in die suidafrikan. Farmwirtschaft und ihr Arbeitskräftepotential der Bantu. Die südafrikan. Farm stellt eine faszinierende Begegnungsstätte zweier grundsätzlich verschiedener Lebens- und Kulturkreise dar. Hier vollzieht sich der Wandel der Lebensform der Bantu vom Squatter (Lohnpächter) bis zum Spezialarbeiter.

Die Kulturlandschaftsgrenze zwischen der individuell geprägten Farmwirtschaft und der kollektiv gebundenen Landnutzung der Bantugebiete ist markant. Zur Förderung der Bantugebiete sind oft Umsiedlungen von Stämmen notwendig, um die weitere Bodenzerstörung durch Uebernutzung zu verhindern, Quellenschutzgebiete zu schaffen und Restbestände an Regenwäldern $z u$ erhalten. Die Arbeit zeigt in wohltuend sachlicher Weise die Entwicklungsprobleme der Bantu-Landwirtschaft. 